Old Dominion University

ODU Digital Commons

Bioelectrics Publications

Frank Reidy Research Center for Bioelectrics

2002

\title{
Mechanism for Membrane Electroporation Irreversibility Under High-Intensity, Ultrashort Electrical Pulse Conditions
}

\author{
R. P. Joshi \\ Old Dominion University \\ K. H. Schoenbach \\ Old Dominion University
}

Follow this and additional works at: https://digitalcommons.odu.edu/bioelectrics_pubs

Part of the Biophysics Commons, $\underline{\text { Cell Biology Commons, and the Electrical and Electronics }}$ Commons

\section{Repository Citation}

Joshi, R. P. and Schoenbach, K. H., "Mechanism for Membrane Electroporation Irreversibility Under High-Intensity, Ultrashort Electrical Pulse Conditions" (2002). Bioelectrics Publications. 242.

https://digitalcommons.odu.edu/bioelectrics_pubs/242

\section{Original Publication Citation}

Joshi, R. P., \& Schoenbach, K. H. (2002). Mechanism for membrane electroporation irreversibility under high-intensity, ultrashort electrical pulse conditions. Physical Review E, 66(5), 052901. doi:10.1103/PhysRevE.66.052901

This Article is brought to you for free and open access by the Frank Reidy Research Center for Bioelectrics at ODU Digital Commons. It has been accepted for inclusion in Bioelectrics Publications by an authorized administrator of ODU Digital Commons. For more information, please contact digitalcommons@odu.edu. 


\title{
Mechanism for membrane electroporation irreversibility under high-intensity, ultrashort electrical pulse conditions
}

\author{
R. P. Joshi and K. H. Schoenbach \\ Department of Electrical and Computer Engineering, Old Dominion University, Norfolk, Virginia 23529-0246
}

(Received 5 July 2002; published 11 November 2002)

\begin{abstract}
An improved electroporation model is used to address membrane irreversibility under ultrashort electric pulse conditions. It is shown that membranes can survive a strong electric pulse and recover provided the pore distribution has a relatively large spread. If, however, the population consists predominantly of larger radii pores, then irreversibility can result. Physically, such a distribution could arise if pores at adjacent sites coalesce. The requirement of close proximity among the pore sites is more easily satisfied in smaller organelles than in outer cell membranes. Model predictions are in keeping with recent observations of cell damage to intracellular organelles (e.g., mitochondria), without irreversible shock at the outer membranes, by a nanosecond, high-intensity electric pulse. This mechanism also explains the greater damage from multiple electric shocks.
\end{abstract}

DOI: 10.1103/PhysRevE.66.052901

PACS number(s): 87.15.Aa, 87.50.Rr, 87.17.Aa

Electroporation in biological cells involves rapid structural rearrangement and formation of pores in the lipid bilayer, in response to an externally applied electric field [1-3]. It is becoming an important technique for inserting biologically active molecules into cells [1-3], for delivery of DNA for gene therapy [4-6], and in the nonthermal killing of microorganisms [7]. Traditionally, most electroporation studies have focused on relatively low external electric fields (less than $1 \mathrm{kV} / \mathrm{cm}$ ), applied over time periods ranging from several tens of microseconds to milliseconds [8]. In a very recent development, electric fields as high as $100 \mathrm{kV} / \mathrm{cm}$ have been used with pulse durations in the nanosecond range $[9,10]$. Advantages in using short electric pulses include (i) negligible thermal heating, (ii) large electric fields and peak powers, with a lower energy input, and (iii) the possibility to select the desired time scales through pulse width manipulation.

The high-intensity electric pulse work has led to the following important observations. (i) It is possible to maintain the integrity of the outer cell membrane despite the high $(\sim 200 \mathrm{kV} / \mathrm{cm})$ electric fields; (ii) multiple pulses do more irreversible damage than single-shot electric shocks; and (iii) irreversible cell damage occurs at the intracellular organelles (e.g., mitochondria), while the outer membranes remain intact. The ability of such short, high-intensity pulses to bring about such preferential intracellular damage is not well understood. Experimental trends do suggest that smaller organelles and membrane-bound substructures are apparently at greater risk of damage in this high-intensity, nanosecond regime. This contribution seeks to address the origin of such intracellular effects through the use of an improved model for electroporation that can provide a potential mechanism for the observed irreversibility at the subcellular level. Although membranes contain proteins and nonlipid components, fluorescent microscopy under applied bias indicates that the electroporated sites are typically much larger in diameter than ion channels. Hence, it appears reasonable to confine the analysis to electroporation through lipid bilayers.

A natural starting point is an evaluation of the prevalent Smoluchowski equation (SE) based electroporation model
$[1,11,12]$. The SE predicts that pores having radius $r$ will expand or contract to minimize the pore energy function $E(r)$ given by $[10,13]$

$$
E(r)=2 \pi \gamma r-\pi \Gamma_{0}+(C / r)^{4}-\left\{\left(\epsilon_{w}-\epsilon_{m}\right) /(2 h)\right\} \pi r^{2} V^{2},
$$

where $\gamma$ is the energy per unit length of the pore perimeter, $\Gamma_{0}$ the energy per unit area of the intact membrane, $h$ the membrane thickness, $V$ the voltage across the membrane, and $\epsilon_{w}$ and $\epsilon_{m}$ the permittivities of water and lipid bilayer, respectively. Most analyses in the literature, except for a few recent reports [14-16], use a constant surface tension parameter $\left(\Gamma=\Gamma_{0}\right)$.

The inadequacies of Eq. (1) are best elucidated by considering the resulting $E(r)$ behavior plotted in Fig. 1. For zero applied voltage, a local minimum in the pore energy is predicted at about $0.8 \mathrm{~nm}$, with a local maximum at about 18 $\mathrm{nm}$. Hence, the model predicts that all pores having radii less than $18 \mathrm{~nm}$ tend to drift toward the dynamically stable radius of $0.8 \mathrm{~nm}$. However, pores with radii exceeding this thresh-

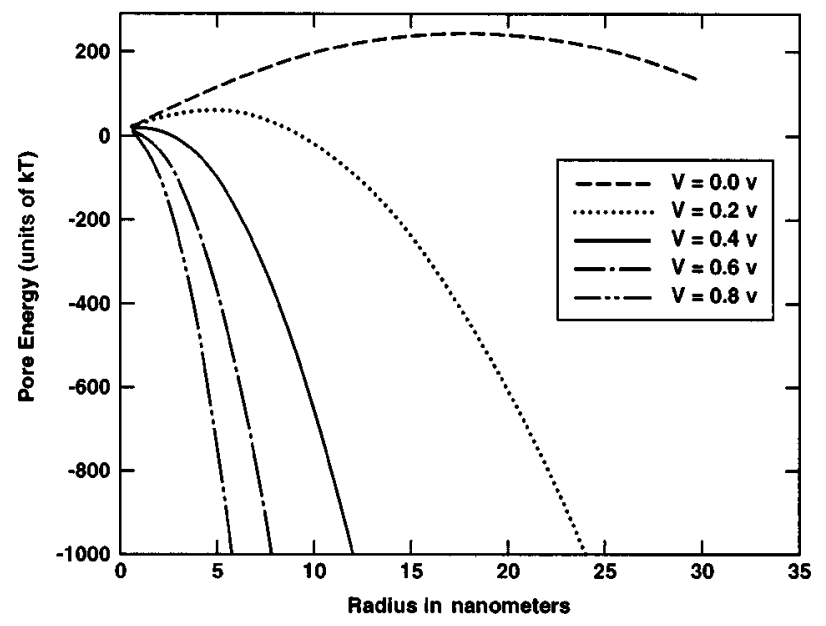

FIG. 1. The pore formation energy $E(r)$ for various membrane voltages. 
old, would drift toward larger values and expand without bound. For $0.4 \mathrm{~V}$ across the cellular membrane, the maximum is virtually eliminated, and represents the minimum voltage for cellular breakdown provided it is applied long enough to enable pores to grow beyond the $18 \mathrm{~nm}$ threshold. This model predicts full cell recovery only if the applied voltage is terminated in time before the critical expansion could take place [17]. At the higher voltages of 0.6 and $0.8 \mathrm{~V}$, the local maximum is not seen, and the pores can potentially expand irreversibly without bound.

The simple energy model of Eq. (1) is unphysical for the following reasons. (i) First, Fig. 1 shows no barrier for $V$ $>0.4 \mathrm{~V}$. However, from experimental data, much higher membrane voltages of about $1.0 \mathrm{~V}$ are required [18] for irreversible breakdown. (ii) Pores with stable diameters up to micrometers in size have been reported [19-21]. This observation is contrary to the theoretical prediction of either complete pore closure or unbounded expansion. (iii) Next, the simple calculation for the voltage dependent term in Eq. (1) does not take into account the finite conductivity of pores, or any charge screening effects. (iv) The use of a constant surface tension $\Gamma_{0}$ becomes questionable. It is expected that the mechanical properties of cells will be modified by deformation caused by the Maxwell stress tensor associated with an external voltage. Experimental results suggest that the tension must naturally be variable, and that its variation facilitates the observed biological activities [22-24]. Since the tension is proportional to the membrane area, at least to first order, it follows that pore formation will lead to variations in $\Gamma$ that are proportional to the square of the pore radius [25]. (v) Next, the formation energy $E(r)$ in Eq. (1) is independent of the pore population and density. However, since the lipid bilayer is essentially elastic and incompressible, it follows that changes in the pore area at constant surfactant molecules must cause changes in the interfacial free energy. This was first discussed many years ago by White [23]. (vi) Finally, the parameters of Eq. (1) are static, and there is no dependence on the dynamical evolution of the pores. Based on the above, $E(r)$ should depend on the pore density $(n)$ to make it self-adjusting in response to $n(r, t)$, without causing uncontrolled pore expansion.

In a recent contribution [14], we developed an improved energy model that includes the dependence of $E(r)$ on pore population and density. Here, we augment this model to include pore-pore interactions and show that pore coalescence in cellular substructures and organelles under ultrashort, high-intensity electric pulse conditions can lead to intracellular instability and damage. As discussed in detail elsewhere [14], the energy equation (1) can be modified to include a variable surface tension, a dynamical dependence on $n(r, t)$, and voltage dependent Born energy corrections [27] arising from the presence of ions in water near pores. The modified pore formation energy is

$$
\begin{aligned}
E(r)= & 2 \pi \gamma r-\left\{\int_{0}^{r} 2 \pi \Gamma_{\text {eff }}\left(A_{P}\left[r^{*}, t\right]\right) r^{*} d r^{*}\right\} \\
& +(C / r)^{4}-\pi\left\{\left(\epsilon_{w}-\epsilon_{m}\right) / h\right\} V^{2} \int_{0}^{r} \alpha^{2}\left(r^{\prime \prime}\right) r^{\prime \prime} d r^{\prime \prime},
\end{aligned}
$$

with $A_{P}\left[r^{*}, t\right]$ being the dynamic pore area, $\Gamma_{\text {eff }}$ a variable membrane tension, and $\alpha$ a pore dependent factor. The dynamic effect of pore area $A_{P}$ on the membrane tension is arrived at by considering a lipid bilayer of total area $A$ and total interfacial energy $W$. The expressions of the energy $W\left(A_{M}=A-A_{P}\right)$ and the tension $\Gamma_{\text {eff }}$ according to [14] are

$$
\begin{aligned}
W\left(A_{M}\right) & =4 \sigma^{\prime} A_{0}+\int_{A_{0}}^{A_{M}} \Gamma_{\mathrm{eff}}\left(A^{\prime}\right) d A^{\prime} \\
& =2 \sigma^{\prime}\left[A-A_{P}+A_{0}^{2} /\left(A-A_{P}\right)\right], \\
\Gamma_{\mathrm{eff}}\left(A_{M}\right) & =\partial\left[2 \sigma^{\prime}\left\{A-A_{P}+A_{0}^{2} /\left(A-A_{P}\right)\right\}\right] / \partial A \\
& =2 \sigma^{\prime}\left[1-\left\{A_{0} /\left(A-A_{P}\right)\right\}^{2}\right],
\end{aligned}
$$

where $\sigma^{\prime}$ is the interfacial energy per area of the hydrocarbon-water interface $\left(\sim 2 \times 10^{-2} \mathrm{~J} \mathrm{~m}^{-2}\right)$ and $A_{0}$ (the equilibrium area) corresponding to minimum total interfacial energy (i.e., $W_{\min }=4 \sigma^{\prime} A_{0}$ ). Area $A$ slightly exceeds the equilibrium level $A_{0}$, and roughly $A / A_{0} \sim 1.0125$, yielding a tension of $10^{-3} \mathrm{~J} \mathrm{~m}^{-2}$ [14]. The effective tension in the presence of pores [i.e., $\left.\Gamma_{\text {eff }}\left(A_{P}\right)\right]$ can then be expressed in terms of the value without pores $\left[\Gamma_{\text {eff }}\left(A_{P}=0\right)\right]$ :

$$
\begin{aligned}
\Gamma_{\text {eff }}\left(A_{P}\right)= & \Gamma_{\text {eff }}\left(A_{P}=0\right)\left[1-\left\{A_{0} / A\right\}^{2}\right] /\left[1-\left\{A_{0} /(A\right.\right. \\
& \left.\left.\left.-A_{P}\right)\right\}^{2}\right] .
\end{aligned}
$$

The pore area in the above expressions represents the average value and hence, is given by the actual SE governed pore density distribution function $n(r, t)$ as

$$
A_{P}(r, t) \sim A_{0}\left[\int_{0}^{r} 2 \pi r^{*} n\left(r^{*}, t\right) d r^{*}\right] .
$$

The $A_{P}(r, t)$ dependent surface tension can become quite important for situations involving transient voltage pulses. In such cases, the voltage could fall to zero quickly (i.e., ultrashort nanosecond pulses), thereby canceling out the electrostatic contribution to $E(r)$. However, the $A_{P}(r, t)$ term would continue to affect dynamical evolution over much longer periods.

The importance of pore distribution on the outcome of an electrical shock is now probed based on the above model. The evolution of $n(r, t)$ into a final steady state can be predicted by starting from an initial condition $n\left(r, t=t_{p}\right)$ subject to the appropriate $E(r)$. For a uniform pore distribution over a finite region of $r$ space (i.e., $r_{1}<r<r_{2}$ ) due to the nearly constant generation rate during the short interval $t_{p}$, i.e., $n\left(r, t=t_{p}\right)=K\left[U\left(r-r_{1}\right)-U\left(r-r_{2}\right)\right]$, where $K$ is a constant and $U$ the Heaviside step function; the ratio $A_{P} / A_{0}$ becomes

$$
\begin{aligned}
A_{P}\left(r^{*}\right) / A_{0} & \sim \int_{r_{1}}^{r_{2}} 2 \pi r^{\prime \prime} K d r^{\prime \prime} \\
& =\pi K\left(r_{2}^{2}-r_{1}^{2}\right) \quad \text { for } r^{*}>r_{2},
\end{aligned}
$$




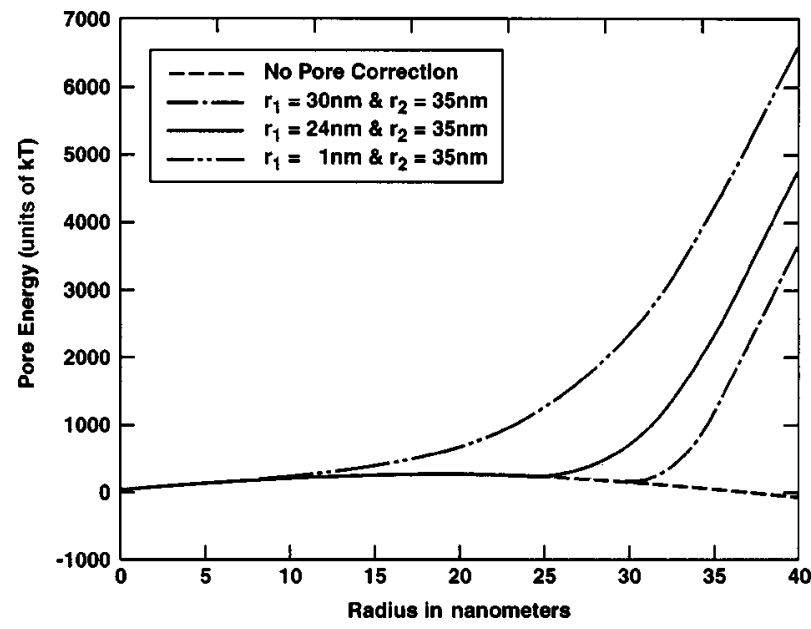

FIG. 2. Energy function $E(r)$ with and without pore corrections for various uniform distributions with pore radii lying between $r_{1}$ and $r_{2}$.

$$
\begin{aligned}
& A_{P}\left(r^{*}\right) / A_{0} \sim \int_{r_{1}}^{r^{*}} 2 \pi r^{\prime \prime} K d r^{\prime \prime} \\
&=\pi K\left(r^{* 2}-r_{1}^{2}\right) \text { for } r_{2}>r^{*}>r_{1}, \\
& A_{P}\left(r^{*}\right) / A_{0} \sim 0 \quad \text { for } r_{1}>r^{*} .
\end{aligned}
$$

Ignoring the steric repulsion terms [the smaller pores cannot lead to any significant affects on $E(r)$ or cause damage, as will be shown], we obtain the following radius dependent expressions for $E(r)$ :

$$
\begin{gathered}
E(r)=2 \pi \gamma r-\pi r^{2} \Gamma_{0} \text { for } r<r_{1}, \\
E(r)=2 \pi \gamma r-\pi r^{2} \Gamma_{0}+\left\{\Gamma_{0} /(1-1 / 1.0125)^{2}\right\} \\
\times\left[K^{-1}\left\{\left[1.0125-\pi K\left(r^{2}-r_{1}{ }^{2}\right)\right]^{-1}-1 / 1.0125\right\}\right. \\
\left.-\pi\left(r^{2}-r_{1}{ }^{2}\right)\right] \quad \text { for } r_{1}<r<r_{2}, \\
E(r)=2 \pi \gamma r-\pi r_{1}{ }^{2} \Gamma_{0}+\left\{\Gamma_{0} /(1-1 / 1.0125)^{2}\right\} \\
\times\left[K^{-1}\left\{\left[1.0125-\pi K\left(r_{2}{ }^{2}-r_{1}{ }^{2}\right)\right]^{-1}-1 / 1.0125\right\}\right. \\
\left.-\pi\left(r_{2}{ }^{2}-r_{1}{ }^{2}\right)\right]-\pi \Gamma_{0}\left(r^{2}-r_{2}{ }^{2}\right) \\
\times\left[\left\{1-\left(1.0125-\pi K\left(r_{2}{ }^{2}-r_{1}{ }^{2}\right)^{-2}\right\} /\left(1-1.0125^{-2}\right)\right]\right. \\
\text { for } r_{2}<r .
\end{gathered}
$$

Plots of $E(r)$ based on the improved model for various values of $r_{1}$ and $r_{2}$ are shown in Fig. 2 for $\gamma=1.8$ $\times 10^{-11} \mathrm{~J} \mathrm{~m}^{-1}, \Gamma_{0}=10^{-3} \mathrm{~J} \mathrm{~m}^{-2}$ (as in Ref. [14]), and $K$ $=0.1 /\left[\pi\left(r_{2}{ }^{2}-r_{1}{ }^{2}\right)\right]$. Also shown for comparison is the curve based on the simple, uncorrected $E(r)$ expression, which has the local maximum at about $18 \mathrm{~nm}$. The following features are evident. (i) For a relatively large spread in pore sizes (curve for $r_{1}=1$ and $r_{2}=35 \mathrm{~nm}$ ), the slope is positive and monotonically increases to higher values with increasing $r$. Hence, upon electric pulse termination, the characteristics of the $E(r)$ curve would force the pores to rapidly shrink in

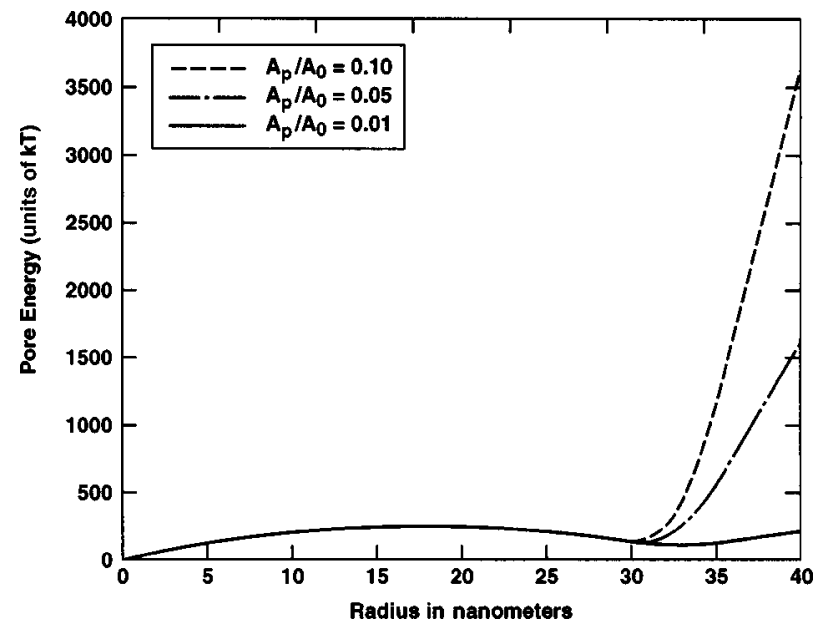

FIG. 3. Energy function with $r_{1}=24$ and $r_{2}=35 \mathrm{~nm}$ for $A_{P} / A_{0}$ ratios of $0.1,0.05$, and 0.01 .

size. Consequently, in spite of a high-intensity field and the large pore population generated, the membrane would naturally be stable due to the dynamic pore-coupled tension, and reversibly recover. (ii) Figure 2 also reveals that no deviation from the uncorrected curve occurs for $r<r_{1}$ [e.g., Eq. (5c)]. Hence, for a narrower spread in the pore distribution (curve with $r_{1}=24$ and $r_{2}=35 \mathrm{~nm}$ ), the energy characteristic retains the $18 \mathrm{~nm}$ local maximum. In addition though, a very shallow energy minimum at about $25 \mathrm{~nm}$, separated from the $18 \mathrm{~nm}$ local maximum, is predicted upon pulse termination. Since the lowest pore radius chosen here is above the $18 \mathrm{~nm}$ maximum, the pores should remain trapped at around $25 \mathrm{~nm}$ even at longer times. This would be in keeping with experimental evidence of long lived pores with radii in the 20-40 $\mathrm{nm}$ range [26]. This simulation case signifies a failure toward pore closure, and a potential for internal damage associated with the loss of membrane regulation. (iii) A similar irreversibility is predicted for the $r_{1}=30$ and $r_{2}=35 \mathrm{~nm}$ curve.

The salient features from Fig. 2 then are the following. (i) Membranes can survive a strong electric pulse and recover provided the pore distribution has a relatively large spread with both small and large sizes present. (ii) If, however, small pores are absent and the population consists predominantly of larger radius pores, then there is a distinct possibility of irreversibility and failure of complete pore closure. Since pores are generated with relatively small radii $[10,13]$, and this is followed by a drift in $r$ space, simplistically then one might expect the distribution to be relatively homogeneous. However, if in addition to a simple expansion in radius pores formed at adjacent sites could coalesce, then a nonuniform distribution would result with few small pores and a non-negligible number with large radii. To facilitate such coalescence, the spatial extent of the membrane surface has to be small, leading to close proximity among the pore sites. Clearly, such a requirement is easier to satisfy for smaller organelles such as mitochondria. This prediction is in keeping with recent observations of damage occurring to intracellular organelles and apoptosis induction by nanosecond, high-intensity pulses, while leaving the outer membrane undamaged [28]. In contrast, conventional theory suggests 
that organelles having larger radii would be most affected by the fields due to a larger membrane voltage. Also, surfaces with high eccentricities (e.g., cigar shaped) would be more susceptible to damage at the "polar caps." Even a relatively modest $A_{P} / A_{0}$ ratio could lead to irreversibility as shown in Fig. 3. Using $A_{P} / A_{0}$ values of $0.1,0.05$, and 0.01 with $r_{1}$ $=24$ and $r_{2}=35 \mathrm{~nm}$, all three curves exhibit a stable minimum beyond $30 \mathrm{~nm}$. Furthermore, the location of this minimum shifts to higher values with decreasing $A_{P} / A_{0}$, indicating that slightly bigger pores, if formed, can endure.

It may be pointed out that a very short electric shock would be insufficient to cause irreversible damage. The rate of change in pore radius is given as $d r / d t=[d E(r) / d r]$ $\times\left\{D /\left[k_{B} T\right]\right\}$. For irreversible breakdown, the pore radius has to grow to at least $r_{\text {crit }}[\sim(18 \mathrm{~nm}) / 2]$ so that upon coalescence the resulting pore radius is above the local energy maximum at $18 \mathrm{~nm}$. Using the hydrophilic pore energy expression, and ignoring the steric repulsion term, one obtains a minimum pulse width $t_{\text {pulse }}$ :

$$
\begin{aligned}
t_{\text {pulse }}= & \left\{\left[k_{B} T\right] / D\right\}\left[2 \pi \sigma+2 \pi a_{p} V^{2}\right]^{-1} \\
& \times \ln \left\{\left[-\gamma+\left(\sigma+a_{p} V^{2}\right) r_{\text {crit }}\right] /\left[-\gamma+\left(\sigma+a_{p} V^{2}\right) r_{\min }\right]\right\} .
\end{aligned}
$$

From Eq. (6), a very short pulse ( $\left.t_{\text {pulse }} \rightarrow 0\right)$ requires a very large membrane voltage $(V \rightarrow \infty)$. Hence, an extremely short pulse cannot be lethal. Since the transmembrane voltage scales with the physical dimension (e.g., the radius $a$ for spherical cells), it would be more difficult to damage smaller entities with a single shot. Repeated pulses, however, would be more harmful because of a greater probability of driving the pores past $r_{\text {crit }}$, and also because newer pores could coalesce with existing ones. This prediction is in keeping with cell viability reductions observed for multiple pulses.

The authors thank H. Hjalmarson and W. Krassowska for useful discussions. This work was sponsored in part by the Air Force Office of Scientific Research.
[1] J. C. Weaver and Y. A. Chizmadzhev, Bioelectrochem. Bioenerg. 41, 135 (1996).

[2] H. Potter, Anal. Biochem. 174, 361 (1988).

[3] S. B. Dev, D. P. Rabussay, G. Widera, and G. A. Hofmann, IEEE Trans. Plasma Sci. 28, 206 (2000).

[4] S. I. Sukharev, V. A. Klenchin, S. M. Serov, L. V. Chernomordik, and Y. Chizmadzhev, Biophys. J. 63, 1320 (1992).

[5] T. E. Zewert, U. F. Pliquett, R. Langer, and J. C. Weaver, Biochem. Biophys. Res. Commun. 212, 286 (1995).

[6] K. Yoshizato, T. Nishi, T. Goto, S. B. Dev, H. Takeshima, T. Kino, K. Tada, T. Kimura, S. Shiraishi, M. Kochi, J. Kuratsu, G. A. Hofmann, and Y. Ushio, Int. J. Oncol. 16, 899 (2000).

[7] A. J. H. Sale and W. A. Hamilton, Biochim. Biophys. Acta 148, 781 (1967).

[8] H. Huelsheger, J. Potel, and E. G. Niemann, Radiat. Environ. Biophys. 20, 53 (1981).

[9] K. H. Schoenbach, S. J. Beebe, and E. S. Buescher, Bioelectromagnetics (N.Y.) 22, 440 (2001).

[10] R. P. Joshi, Q. Hu, R. Aly, K. H. Schoenbach, and H. P. Hjalmarson, Phys. Rev. E 64, 11913 (2001).

[11] V. F. Pastushenko, Yu A. Chhizmadzhev, and V. B. Arakelyan, Bioelectrochem. Bioenerg. 6, 53 (1979).

[12] A. Barnett and J. C. Weaver, Bioelectrochem. Bioenerg. 25, 163 (1991).

[13] J. C. Neu and W. Krassowska, Phys. Rev. E 59, 3471 (1999).
[14] R. P. Joshi, Q. Hu, K. H. Schoenbach, and H. P. Hjalmarson, Phys. Rev. E 65, 041920 (2002).

[15] H. Isambert, Phys. Rev. Lett. 80, 3404 (1998).

[16] O. Sandre, L. Mareaux, and F. Brochard-Wyart, Proc. Natl. Acad. Sci. U.S.A. 96, 10591 (1999); F. Brochard-Wyart, P. G. de Gennes, and O. Sandre, Physica A 278, 32 (2000).

[17] R. P. Joshi and K. H. Schoenbach, Phys. Rev. E 62, 1025 (2000).

[18] W. Meier, A. Graff, A. Diederich, and M. Winterhalter, Phys. Chem. Chem. Phys. 2, 4559 (2000).

[19] S. E. Feller and R. W. Pastor, Biophys. J. 71, 1350 (1996).

[20] S. I. Sukharev, P. Blount, B. Martinac, F. R. Blattner, and C. Kung, Nature (London) 368, 265 (1994).

[21] T. Benachir and M. Lafleur, Biophys. J. 70, 831 (1996).

[22] H. Isambert, Phys. Rev. Lett. 80, 3404 (1998).

[23] S. H. White, Biophys. J. 14, 155 (1974).

[24] D. V. Zhelev and D. Needham, Biomembranes 1147, 89 (1993).

[25] O. Sandre, L. Mareaux, and F. Brochard-Wyart, Proc. Natl. Acad. Sci. U.S.A. 96, 10591 (1999).

[26] D. C. Chang and T. S. Reese, Biophys. J. 58, 1 (1990).

[27] V. F. Pastushenko and Yu A. Chhizmadzhev, Biofizika 28, 1036 (1983); 27, 475 (1982).

[28] K. H. Schoenbach, S. J. Beebe, and E. S. Buescher, Bioelectromagnetics (N.Y.) 22, 440 (2001). 